\title{
El diálogo en la relación médica odontólogo-paciente desde la mirada de Gadamer: caso mucositis oral
}

\begin{abstract}
The dialogue in the doctor (dentist)-patient relationship from Gadamer's view: case of oral mucositis
\end{abstract}

\section{O diálogo na relação médico-dentista na perspectiva de Gadamer: caso de mucosite oral}

\begin{abstract}
Alejandra Sosa Gómez
Instituto Politécnico Nacional, Centro Interdisciplinario de Ciencias de la Salud Unidad Santo Tomás, Centro de Investigaciones Económicas, Administrativas y Sociales, México alejandrasosagmz@gmail.com https://orcid.org/0000-0002-2645-3863

Alma Alicia Benítez Pérez * Instituto Politécnico Nacional, Centro de Estudios Científicos y Tecnológicos 11 "Wilfrido Massieu", Centro de Investigaciones Económicas, Administrativas y Sociales, México albenper@gmail.com https://orcid.org/0000-0001-9213-0379

*Autor de correspondencia
\end{abstract}

\section{Resumen}

Objetivo: Indagar sobre el diálogo en la relación médica odontólogo-paciente, particularmente en las mujeres con tratamiento de quimioterapia por cáncer de mama: caso mucositis oral en una institución especializada. Materiales y métodos: Se realizó un estudio prospectivo, descriptivo y transversal, para lo cual se diseñó un cuestionario basado en la cédula PRO-CTCAETM (siglas en inglés) atendiendo principalmente al cuidado bucal, así como a la asistencia y la atención por el odontólogo, lo que contribuyó a conocer la relación médica odontólogo-paciente con base en el diálogo para favorecer la calidad de vida de las pacientes. Se contó con 50 participantes cuyas edades oscilaron entre los 31 y 


\section{Revista Iberoamericana \\ de las Ciencias Sociales y Humanísticas}

ISSN: $2395-7972$

75 años. Resultados: Los resultados más significativos revelan que $68 \%$ no asiste a consulta odontológica antes de iniciar la quimioterapia y $34 \%$ presentan úlceras en la mucositis oral, lo que limita funciones básicas como comer, beber y comunicarse. Conclusiones: Los hallazgos muestran la necesidad de impulsar el acompañamiento de un equipo multidisciplinario durante la quimioterapia, donde la participación del odontólogo debe ser esencial para la atención bucodental. Lo anterior desde la hermenéutica filosófica propuesta por Gadamer, cuyo acercamiento permite fortalecer, desde una visión holística, la calidad de vida bucal del paciente por efectos secundarios (mucositis oral) de la quimioterapia.

Palabras clave: cáncer de mama, diálogo, hermenéutica filosófica, mucositis oral, quimioterapia.

\section{Abstract}

Objective: To investigate the dialogue in the doctor (dentist)-patient relationship, particularly in women with chemotherapy for breast cancer; case oral mucositis in a specialized institution. Material and methods: A prospective, descriptive, cross-sectional study was carried out, for which a questionnaire based on the PRO-CTCAE TM card (acronym in English) was designed, mainly attending to oral care, as well as assistance and attention by the dentist, which contributed to knowing the doctor (dentist)-patient relationship based on dialogue to promote the quality of life of patients. There were 50 participants whose ages ranged from 31 to 75 years. Results: The most significant results show that $68 \%$ do not attend a dental consultation before starting chemotherapy and $34 \%$ of patients present ulcers in oral mucositis, limiting basic functions such as eating and drinking, which affects isolation. Conclusions: The findings show the need to promote the accompaniment of a multidisciplinary team during chemotherapy, the participation of the dentist being irrefutable in oral care from the philosophical hermeneutics proposed by Gadamer, with a holistic vision whose approach allows to strengthen the Oral Quality of Life of the patient by side effects of chemotherapy, case, oral mucositis.

Keywords: breast cancer, dialogue, philosophical hermeneutics, oral mucositis, chemotherapy. 


\section{Revista Iberoamericana \\ de las Ciencias Sociales y Humanísticas}

\section{Resumo}

Objetivo: Investigar o diálogo na relação médico-dentista, principalmente em mulheres em tratamento quimioterápico para câncer de mama: caso de mucosite oral em instituição especializada. Materiais e métodos: Foi realizado um estudo prospectivo, descritivo e transversal, para o qual foi elaborado um questionário baseado na ficha PRO-CTCAE TM (sigla em inglês), principalmente no atendimento à higiene bucal, bem como assistência e cuidados aos o paciente .dentista, o que contribuiu para o conhecimento da relação médicodentista baseada no diálogo para a promoção da qualidade de vida dos pacientes. Havia 50 participantes com idades variando entre 31 e 75 anos. Resultados: Os resultados mais significativos revelam que $68 \%$ não vão à consulta odontológica antes de iniciar a quimioterapia e 34\% apresentam úlceras na mucosite oral, o que limita funções básicas como comer, beber e se comunicar. Conclusões: Os achados apontam para a necessidade de se promover o apoio de uma equipe multiprofissional durante o tratamento quimioterápico, onde a participação do cirurgião-dentista deve ser imprescindível para os cuidados bucais. O exposto desde a hermenêutica filosófica proposta por Gadamer, cuja abordagem permite fortalecer, numa perspectiva holística, a qualidade de vida oral do paciente devido aos efeitos colaterais (mucosite oral) da quimioterapia.

Palavras-chave: câncer de mama, diálogo, hermenêutica filosófica, mucosite oral, quimioterapia.

Fecha Recepción: Enero 2021

Fecha Aceptación: Junio 2021

\section{Introducción}

Para Gadamer (2005) el diálogo es aquella forma del lenguaje en la que conectamos creando palabras tejidas llevadas por la cosa o situación hermenéutica para llegar a la fusión de horizontes donde es posible que los dialogantes se interesen por la opinión del otro como un acto de razón (Viveros, 2019). En otras palabras, la comprensión implica ponerse de acuerdo en alguna cosa, lo cual solo sucede cuando se tiene la capacidad de dialogar.

En el diálogo, existe un intercambio de mensajes generados por un proceso de preguntas y respuestas; en este sentido, Gadamer (2005) compara el significado de la pregunta con la idea de "abrir", esto es, estar dispuesto a la apertura para la comprensión de una situación o cosa. 


\section{Revista Iberoamericana de las Ciencias Sociales y Humanísticas}

ISSN: $2395-7972$

Gadamer (1993) describe el círculo hermenéutico como un proceso estructurado donde la regla anticipa que el todo se debe entender desde lo individual, y viceversa. La hermenéutica moderna, con antecedentes de la retórica antigua, hace referencia al arte de hablar y comprender basada en una relación circular con tres pilares cuyo punto de partida es el diálogo; estos son interpretación, comprensión y aplicación. La interpretación se caracteriza porque el intérprete lleva el tema en su contexto y con ello tiene una mejor verdad textual, aunque muchas veces se trata de una aproximación (Beuchot, 2016). La comprensión es característica de la persona en su convivencia con los demás y actúa especialmente por vía del lenguaje y del diálogo. Por último, la aplicación permite la apertura a nuevos conocimientos e investigaciones bajo una comprensión de mayor profundidad, derivada de las preguntas formuladas para alcanzar la fusión de horizontes.

Ahora bien, el círculo hermenéutico se ha trasladado a distintas disciplinas, incluso al campo de la medicina. Un ejemplo es el trabajo de Austgard (2012), quien se apoyó en la hermenéutica para desarrollar un plan de investigación en la ciencia de la enfermería para valorar el lenguaje escrito textos a partir de su comprensión, interpretación y aplicación.

Asimismo, Posadas (2009) considera que el diagnóstico es un acto hermenéutico, pues su punto de partida se basa en un diálogo que permite recabar elementos para una historia clínica, así como en un examen físico completo y el uso racional de exámenes complementarios (p. ej., pruebas de laboratorio y gabinete).

En tal sentido, la relación médica odontólogo-paciente constituye el núcleo de la medicina. De hecho, esta se sustenta en una relación comunicativa y participativa en pro de mejorar el aspecto ético (Ávila, 2017) para ir más allá de establecer el diagnóstico y tratamiento. Para ello, es necesario comprender y respetar al paciente como un ser humano con sus propias particularidades, creencias, actitudes y conocimientos diferentes a los del médico. En esta posición, el médico puede lograr que el paciente sea reconocido y, por tanto, mejore su calidad de vida y recupere su salud.

En el marco de la hermenéutica, la dinámica de la relación médica odontólogopaciente parte de un diálogo que inicia escuchando a este último. Al respecto, Ceriani (2017) cita a William Osler con una frase muy importante: "Escuchen a sus pacientes, les estarán diciendo su diagnóstico" (p. 107). Por eso, uno de los objetivos centrales de la medicina es atender las necesidade3s de los pacientes durante el acompañamiento de la enfermedad. La comprensión, en la relación médica odontólogo-paciente, constituye un pilar en el restablecimiento de la condición de salud y la mejora en la calidad de vida. Por 


\section{Revista Iberoamericana \\ de las Ciencias Sociales y Humanísticas}

ISSN: $2395-7972$

ende, el médico debe asegurarse que los pacientes, desde sus posibilidades de comprensión, entiendan lo que les sucede, así como el propósito de su tratamiento.

En ese contexto que se establece, la disposición de los dialogantes contempla la opinión del otro como un acto de razón, lo cual se refleja en las preguntas y respuestas que van emergiendo. Así, el paciente entra en diálogo con el médico y asume que existe un interés no ceñido en la sintomatología para comprender su situación.

Retomando la visión de Gadamer (2001), en la humanización de la medicina se trata de ampliar los horizontes de comprensión mediante el diálogo, por lo cual es necesaria la apreciación holística de los pacientes para llegar a la comprensión del estado de salud. Si bien en el ámbito médico la dimensión física se considera punto de partida para conocer la enfermedad, es necesario resaltar que los pacientes son seres sociales y que los daños orgánicos repercuten en las diferentes esferas de la vida de una persona: lo físico, lo social, lo psicológico y lo ambiental (Fleury y Lana da Costa, 2004).

La dimensión física hace referencia a la capacidad de una persona para realizar las actividades diarias, tales como el desplazamiento, la destreza, el cuidado personal y la deambulación. La social se vincula con la manera en que las personas interactúan con el medio, ya sea la escuela, el trabajo o la familia. Por su parte, la psicológica se relaciona a las emociones, sentimientos, estado de ánimo, entre otros, mientras que la ambiental se asocia con la percepción sobre el ámbito en el que se vive.

Al considerar esas dimensiones, en la vida de una persona con diagnóstico de cáncer de mama, es posible reconocer un notable deterioro en su calidad de vida. Al inicio, los trastornos físicos cobran importancia por los daños orgánicos y con el paso del tiempo las repercusiones invaden el resto de las esferas, lo cual se convierte en un factor determinante en el proceso de recuperación. De acuerdo con Taichman, Poznack y Inglehart (2018), las pacientes pueden llegar a abandonar los tratamientos debido a los efectos secundarios de la quimioterapia, o bien por la falta de recursos económicos o las limitaciones en la actividad laboral. Al respecto, Walsh (2010) señala que la economía representa una preocupación trascendental para tratar el cáncer y todas sus complicaciones.

El amplio abanico de las dimensiones influye en quien padece la enfermedad, por lo que es necesaria una postura que haga frente al diálogo; en respuesta a ello, la hermenéutica filosófica — desde la mirada de Gadamer (2001) — constituye una de las herramientas para optimizar la comunicación con las pacientes que padecen los efectos secundarios por la quimioterapia. Desde esta visión, Gadamer aboga por la vinculación de la ciencia moderna, 


\section{Revista Iberoamericana}

de las Ciencias Sociales y Humanísticas

ISSN: 2395 - 7972

empírica y basada en evidencia matematizable con las ciencias del espíritu, las cuales se ocupan de las maneras en que sentimos, experimentamos y hacemos conciencia de los fenómenos humanos. Esto se puede complementar a través de una mentalidad hermenéutica capaz de establecer un diálogo entre las dimensiones física (derivada de la ciencia empírica), social y psicológica (Viveros, 2019).

El valor de la hermenéutica como recurso para la comprensión del diálogo médico odontólogo-paciente se traza bajo dos fines: el primero relacionado con el diagnóstico de la condición o enfermedad de un paciente (dialéctica del conocimiento clínico-paciente); el otro, para guiar al paciente durante la quimioterapia y ofrecer el acompañamiento necesario para cuando se presenten daños por los efectos secundarios, entre los que se destacan aquellos vinculaos con la salud oral, como la mucositis oral, las alteraciones en el gusto y la disminución de la saliva (xerostomía) (Barroso-Sousa et al., 2013; Jensen et al., 2008).

\section{Mucositis}

La mucositis oral es uno de los efectos secundarios en las pacientes con procedimiento de radioterapia y quimioterapia. Se caracteriza por la presencia de úlceras dolorosas que pueden afectar la totalidad de las estructuras blandas de la boca, de ahí que los pacientes aumenten el consumo de analgésicos narcóticos e incrementen el número y el tiempo de las hospitalizaciones. En esta condición, el aporte nutricional es invasivo, lo que impacta en todos los ámbitos de la vida (Senkus et al., 2013).

Taichman et al. (2015) afirman que las complicaciones trascienden la fase de la quimioterapia del cáncer, puesto que de manera tardía también afectan la calidad de vida al ser alterada la salud periodontal por la reducción del flujo salival (hiposalivación); en este caso, es posible el incremento en la gingivitis y caries dental, así como disestesia oral y disgeusia (alteración en el sentido del gusto).

Asimismo, la afectación en la dimensión física desencadena repercusiones en el resto de las dimensiones, pues las úlceras, la hiposalivación y la alteración en el gusto deterioran las funciones básicas, lo cual se vuelve más crítico en aquellos casos con problemas bucodentales previos.

Por eso, en el referido tratamiento con quimioterapia se debe advertir a los pacientes de los efectos bucales secundarios y comunicarlo al odontólogo para que realice una valoración dental (Taichman et al., 2018; Walsh, 2010). Otro ejemplo son las pacientes tratadas con terapia antiestrogénica, las cuales pueden presentar grandes niveles de 


\section{Revista Iberoamericana \\ de las Ciencias Sociales y Humanísticas}

ISSN: $2395-7972$

depresión, así como síntomas músculo-esqueléticos y fatiga, lo que puede disminuir la habilidad y disposición para mantener una óptima salud bucal con acciones tan simples como el cepillado dental (Taichman et al., 2018). Cabe subrayar que los fármacos indicados no se deben suspender; por el contrario, las pacientes deben adherirse a los protocolos para evitar la interferencia en el resto de las dimensiones, con lo cual se procura mejorar su calidad de vida.

En los ejemplos señalados, la prevención con la atención bucodental previa o posterior a la quimioterapia reducirá las complicaciones. Por ello, el acompañamiento de las pacientes y el diálogo abierto permitirán monitorear constantemente la evolución, de ahí que se haga énfasis en la aplicación de un método multidisciplinario de atención al paciente (Seiler, Kosse, Loibl y Jackisch, 2014).

Ahora bien, centrándonos en el tema de la mucositis oral por quimioterapia de mama, cabe subrayar que hasta la fecha dicha línea de investigación se encuentra en desarrollo. Por tal motivo, en el presente trabajo se procura identificar los parámetros para el análisis del conocimiento que tienen las pacientes sobre los efectos secundarios de la quimioterapia en torno a la mucositis oral. Para esto, se ha considerado al paciente desde una concepción holística (que incluye lo físico, social y psicológico) en el marco del diálogo hermenéutico.

Dicho esto, en esta investigación se plantea un acercamiento al diálogo para mejorar la relación médica odontólogo-paciente durante el acompañamiento de la quimioterapia. Por ende, el preámbulo de dicha meta parte del diagnóstico que inicia con la apertura al conocimiento del otro, lo cual ofrece un panorama más amplio acerca de la comprensión que tienen las pacientes sobre la quimioterapia para tratar el cáncer de mama y sobre los efectos bucales de dicha intervención con la finalidad de contribuir a mejorar su calidad de vida bucal.

Desde esta postura, la intención de este trabajo fue establecer los parámetros de

análisis para fundamentar y dilucidar los elementos frágiles en las pacientes, así como situar el punto de anclaje para establecer el diálogo y, en consecuencia, proporcionar el apoyo y guía durante la quimioterapia. 


\section{Materiales y métodos}

El presente es un estudio de tipo prospectivo, descriptivo y transversal, pues se consultaron los registros a partir de la cédula para la recolección de los resultados informados por el paciente conforme a los criterios de terminología común para eventos adversos —en inglés se conoce como Patient-reported outcomes - Common Terminology Criteria for Adverse Events (PRO-CTCAETM) - .

La muestra estuvo constituida por 50 pacientes que acudieron a consulta externa de un centro de atención especializado en cáncer de mama, en el turno matutino, durante el periodo de septiembre a octubre de 2019. La cédula de recolección de datos se aplicó a las pacientes al salir de su consulta. Se consideraron las variables demográficas de las participantes, las cuales fueron mujeres con un rango de edad de 31 a 75 años, quienes aceptaron participar de manera voluntaria.

Las variables dependientes fueron las siguientes: pacientes que recibieron información sobre los efectos secundarios; personal de salud que informó sobre los efectos secundarios; trato especial en el cuidado bucal; asistencia y atención por el odontólogo. Estas variables contribuyen a conocer la relación médico-paciente, lo que permitirá impulsar el diálogo para favorecer la calidad de vida de las pacientes con quimioterapia por cáncer de mama al reducir y atender la mucositis oral como uno de los principales efectos secundarios.

\section{El instrumento de medición}

Se utilizó la cédula de recolección de datos autoaplicables PRO-CTCAETM, la cual fue acotada a las preguntas relacionadas con la mucositis oral. Para su aplicación se agregó el número de folio, instrucciones de llenado y datos generales (edad, escolaridad y ocupación).

Cabe señalar que dicho instrumento estuvo constituido por preguntas relacionadas con las dimensiones física, psicológica y social (Bash et al., 2014). El cuestionario permite conocer si las pacientes recibieron información del personal de salud sobre los efectos secundarios bucales de la quimioterapia, así como de la existencia o ausencia del acompañamiento en dicho proceso.

La cédula se conformó por 21 ítems (sin considerar los datos generales). Para el análisis de la información las preguntas se agruparon en las siguientes categorías: a) datos generales de las pacientes (edad, escolaridad, ocupación); b) conocimiento de los 


\section{Revista Iberoamericana \\ de las Ciencias Sociales y Humanísticas}

ISSN: $2395-7972$

medicamentos de la quimioterapia, consulta odontológica y atención dental (5 ítems), y c) manifestaciones bucales (mucositis oral) por efectos secundarios a la quimioterapia (16 ítems), con alteraciones en las dimensiones física y social.

De esta manera, se analizaron los tres grupos y sus ítems representativos. Asimismo, se consideraron todos los datos de los grupos A y B con el propósito de conocer la actividad laboral, la escolaridad y la edad como posibles variables relacionadas con el acceso a la información sobre su condición médica y los efectos secundarios en la cavidad bucal, así como la interacción con el odontólogo y su intervención en el apoyo de las pacientes. Del grupo C se observó lo siguiente: la severidad de las úlceras bucales después de iniciar la quimioterapia, las úlceras bucales y la interferencia con el habla, la comunicación y la ingesta de alimentos o bebidas, así como la suspensión de la quimioterapia (ítems 5, 18, 19 y 20) vinculada con las dimensiones física y social.

\section{Procedimiento de recolección de datos}

La recolección de los datos mediante la cédula PRO-CTCAETM favorece el anonimato y la libertad de expresar las respuestas, con la posibilidad de que el entrevistador, dispuesto al diálogo, pueda aclarar dudas y retroalimentar las inquietudes del paciente. En ese marco, se explicó a las participantes el objetivo del estudio y la confidencialidad de la información, así como el anonimato de la cédula, además de reiterar el apoyo o aclaración de dudas.

La cédula fue contestada de manera individual, y una vez recabada la información, las respuestas se vaciaron en una hoja de cálculo Excel de Microsoft; en el análisis de los datos se empleó el paquete estadístico SPSS (versión 22) para el análisis de las variables desde la visión de la hermenéutica filosófica.

Teniendo en cuenta lo anterior, las consideraciones éticas de esta investigación se fundamentaron en tres aspectos: primero, en las cédulas aplicadas no se solicitó el nombre del participante y se reiteró que la información recabada sería utilizada para fines del presente estudio con carácter confidencial y anónimo; segundo, el diseño de una carta de consentimiento informado, con copia para las pacientes, con explicación sobre los detalles del estudio, la duración y los beneficios de las actividades; y tercero, el estudio se realizó en la Ciudad de México, de septiembre a octubre del 2019, bajo la aprobación del Comité de Ética del CICS-UST (Registro número: CONBIOÉTICA-09-CEI-019-20170731). 


\section{Resultados}

Se entrevistaron a 50 pacientes, con un rango de edad de 31 a 75 años (promedio de 51.8 años). Respecto a la escolaridad, del total de entrevistadas, $30 \%$ cuenta con estudios de secundaria, $28 \%$ primaria, $18 \%$ licenciatura, $8 \%$ no especificó, $6 \%$ estudios de bachillerato y ninguna señaló estudios de maestría o doctorado. Respecto a la ocupación, 76 $\%$ se encargan de las labores domésticas, mientras que $24 \%$ de otras ocupaciones y $6 \%$ son jubiladas.

En cuanto a haber recibido información de los efectos secundarios de la quimioterapia, $66 \%$ respondió afirmativamente, mientras que $34 \%$ no fueron informadas. Las encuestadas reportaron que los encargados de informar sobre los efectos secundarios fueron el oncólogo (48\%), el médico general (10\%) y las enfermeras (8\%). Como puede observarse, ninguna paciente reportó haber tenido diálogo con el odontólogo.

En relación con las preguntas correspondientes al conocimiento de los medicamentos de la quimioterapia, la consulta odontológica y la atención dental, los resultados se presentan en la tabla 1. 


\section{Revista Iberoamericana \\ de las Ciencias Sociales y Humanísticas}

ISSN: $2395-7972$

Tabla 1. El conocimiento de los medicamentos de la quimioterapia, la consulta odontológica y la atención dental

\begin{tabular}{|c|c|c|c|c|c|}
\hline Respuesta & $\begin{array}{c}\text { Pacientes que } \\
\text { recuerdan el } \\
\text { nombre de los } \\
\text { medicamentos } \\
\text { de la } \\
\text { quimioterapia } \\
\text { n }(\%)\end{array}$ & $\begin{array}{c}\text { Antes de } \\
\text { iniciar la } \\
\text { quimioterapia } \\
\text { acudieron a } \\
\text { consulta } \\
\text { odontológica } \\
\text { n }(\%)\end{array}$ & $\begin{array}{c}\text { Durante la } \\
\text { quimioterapia } \\
\text { recibieron } \\
\text { tratamiento } \\
\text { dental } \\
\text { n }(\%)\end{array}$ & $\begin{array}{c}\text { Durante la } \\
\text { quimioterapia } \\
\text { recibieron } \\
\text { trato especial } \\
\text { en el cuidado } \\
\text { bucal } \\
\mathrm{n}(\%)\end{array}$ & $\begin{array}{c}\text { Especifique } \\
\text { el cuidado } \\
\text { especial } \\
\text { n }(\%)\end{array}$ \\
\hline Sí & $\begin{array}{c}6 \\
(12 \%)\end{array}$ & $\begin{array}{c}16 \\
(32 \%)\end{array}$ & $\begin{array}{c}9 \\
(18 \%)\end{array}$ & $\begin{array}{c}22 \\
(44 \%)\end{array}$ & $\begin{array}{c}\text { Cepillado } \\
\text { dental: } 11 \\
(22 \%) \\
\text { Enjuague: } \\
10(20 \%) \\
\text { Uso de } \\
\text { pasta } \\
\text { dental:1 } \\
(2 \%) \\
\text { Cuidado } \\
\text { dental: } 1(2 \\
\%)\end{array}$ \\
\hline No & $\begin{array}{c}43 \\
(86 \%)\end{array}$ & $\begin{array}{c}34 \\
(68 \%)\end{array}$ & $\begin{array}{c}41 \\
(82 \%)\end{array}$ & $\begin{array}{c}28 \\
(56 \%)\end{array}$ & NA \\
\hline No contestó & $\begin{array}{c}1 \\
(2 \%)\end{array}$ & 0 & 0 & 0 & $\mathrm{NA}$ \\
\hline Total & $\begin{array}{c}50 \\
(100 \%)\end{array}$ & $\begin{array}{c}50 \\
(100 \%)\end{array}$ & $\begin{array}{c}50 \\
(100 \%)\end{array}$ & $\begin{array}{c}50 \\
(100 \%)\end{array}$ & $\mathrm{NA}$ \\
\hline
\end{tabular}

Fuente: Elaboración propia

Con respecto al primer tema, se observó un acentuado desconocimiento por parte de las pacientes sobre los medicamentos de la quimioterapia: 86 \% reportó no saberlo, $12 \%$ recordó el nombre de los fármacos y una persona no contestó. Como puede notarse, pese a que más de la mitad de las pacientes recibieron información sobre los efectos secundarios, desconocen los medicamentos de su quimioterapia.

Por otra parte, $68 \%$ refirió la nula asistencia a la consulta odontológica antes de iniciar la quimioterapia y, por tanto, la ausencia de tratamiento dental. Las pacientes que reportaron la asistencia a consulta odontológica durante la quimioterapia (32\%) recibieron información general sobre la higiene dental, la cual consistió principalmente en el cepillado dental y uso de enjuagues convencionales debido a que no existe un protocolo de atención 


\section{Revista Iberoamericana \\ de las Ciencias Sociales y Humanísticas}

ISSN: 2395 - 7972

multidisciplinaria que permita acompañar a las pacientes no solo para atender problemas bucodentales emergentes, sino incluso para prevenirlos y monitorear constantemente las medidas de higiene bucal.

En cuanto al tipo de cuidado bucal que recibieron las pacientes durante la quimioterapia, el cepillado dental se limitó a 11 casos (22 \%), enjuague bucal a 10 casos $(20 \%)$ y el uso de la pasta dental a un caso. Cabe mencionar que este tipo de cuidado se refiere al dental, y no a los efectos secundarios de la quimioterapia, como la mucositis oral.

En la tabla 2 se compara la severidad de las úlceras bucales después de iniciar la quimioterapia: $54 \%$ de las pacientes reportaron que no tenían úlceras bucales, mientras que $46 \%$ presentaban úlceras leves o muy severas. Es importante mencionar la posibilidad de presentar las úlceras si continúan con la quimioterapia, pues su presencia depende de varios factores, como el tiempo de tratamiento y de la combinación de los fármacos, entre otros no considerados para este estudio.

Tabla 2. Severidad de las úlceras bucales después de iniciar la quimioterapia

\begin{tabular}{|c|c|c|c|c|c|}
\hline $\begin{array}{c}\text { Ninguna } \\
\mathrm{n}(\%)\end{array}$ & $\begin{array}{c}\text { Leve } \\
\mathrm{n}(\%)\end{array}$ & $\begin{array}{c}\text { Moderada } \\
\mathrm{n}(\%)\end{array}$ & $\begin{array}{c}\text { Severa } \\
\mathrm{n}(\%)\end{array}$ & $\begin{array}{c}\text { Muy severa } \\
\mathrm{n}(\%)\end{array}$ & $\begin{array}{c}\text { Total } \\
\mathrm{n}(\%)\end{array}$ \\
\hline 27 & 11 & 7 & 2 & 3 & 50 \\
$(54 \%)$ & $(22 \%)$ & $(14 \%)$ & $(4 \%)$ & $(6 \%)$ & $(100 \%)$ \\
\hline
\end{tabular}

Fuente: Elaboración propia

Por otra parte, $88 \%$ de las pacientes manifestaron que no suspendieron la quimioterapia, mientras que el restante $12 \%$ no contestó. En $34 \%$ de las pacientes impidieron funciones básicas como el comer y beber, así como el habla o la comunicación (tabla 3). La adherencia al tratamiento es uno de los aspectos más importantes, pues condiciona la recuperación de la salud; además, el acompañamiento y la asistencia a las pacientes pueden evitar la reducción en la calidad de vida, aunque esto también implica un aumento en el costo del tratamiento, lo cual es un tema que debe atenderse como parte del proceso de humanización de la medicina. 
Revista lberoamericana

de las Ciencias Sociales y

Humanísticas

ISSN: 2395 - 7972

Tabla 3. Las úlceras bucales y la interferencia con el habla, la comunicación y la ingesta de alimentos o bebidas, así como la suspensión de la quimioterapia

\begin{tabular}{|l|c|c|c|}
\hline \multicolumn{1}{|c|}{ Respuesta $(\%)$} & $\begin{array}{c}\text { Las úlceras bucales } \\
\text { impidieron el habla o la } \\
\text { comunicación con otras } \\
\text { personas } \\
\mathrm{n}(\%)\end{array}$ & $\begin{array}{c}\text { Las úlceras bucales } \\
\text { impidieron comer } \\
\text { algún tipo de alimento } \\
\text { o bebida } \\
\mathrm{n}(\%)\end{array}$ & $\begin{array}{c}\text { Ha tenido que } \\
\text { suspender la } \\
\text { quimioterapia por las } \\
\text { úlceras bucales } \\
\mathrm{n}(\%)\end{array}$ \\
\hline $\mathrm{Si}$ & 7 & 10 & 0 \\
\hline No & $(14 \%)$ & $40 \%)$ & 44 \\
\hline No \\
contestó
\end{tabular}

Fuente: Elaboración propia

En la tabla 4 se aprecia la instancia médica para la atención en caso de presentar úlceras bucales, así como los diferentes motivos para acudir. Cabe mencionar que $50 \%$ de las pacientes eligieron al centro especializado en cáncer de mama como la instancia de mayor confianza debido al servicio que otorga y a lo económico de sus tratamientos. 
Revista Iberoamericana

de las Ciencias Sociales y

Humanísticas

ISSN: $2395-7972$

Tabla 4. Motivos para acudir a una instancia médica en caso de presentar úlceras bucales

\begin{tabular}{|c|c|c|}
\hline $\begin{array}{c}\text { Instancia médica a la que } \\
\text { acudiría en caso de presentar } \\
\text { úlceras bucales }\end{array}$ & $\begin{array}{c}\text { Pacientes } \\
\mathrm{n}(\%)\end{array}$ & $\begin{array}{c}\text { Motivos } \\
\text { n }(\%)\end{array}$ \\
\hline $\begin{array}{l}\text { Centro especializado en cáncer } \\
\text { de mama }\end{array}$ & $25(50 \%)$ & $\begin{array}{c}\text { Confianza: } 19(38 \\
\%)\end{array}$ \\
\hline Dentista & $13(26 \%)$ & Servicio: $13(26 \%)$ \\
\hline Médico particular & $5(10 \%)$ & Cercania: $9(18 \%)$, \\
\hline Centro de salud & $4(8 \%)$ & Recomendación: 3 \\
\hline IMSS & $1(2 \%)$ & $(6 \%)$ \\
\hline SESA & $1(2 \%)$ & $\begin{array}{c}\text { Rapidez: } 1 \text { (2 \%) } \\
\text { No contestó: } 3 \text { (6 } \\
\%)\end{array}$ \\
\hline No contestó & $1(2 \%)$ & \\
\hline Total & $50(100 \%)$ & \\
\hline
\end{tabular}

Fuente: Elaboración propia

El diálogo con el odontólogo permite a las pacientes conocer la participación de otros profesionales, además del oncólogo o la enfermera, para sumar esfuerzos encaminados a mejorar su calidad de vida en la medida de no ver mermadas las funciones básicas de toda persona, como comer, hablar y comunicarse.

\section{Discusión}

De acuerdo con los resultados obtenidos, la relación médica odontólogo-paciente es limitada, pues el oncólogo es quien se encarga de informar sobre el padecimiento y tratamiento de quimioterapia (48\%). De hecho, en caso de presentar efectos secundarios, como la mucositis oral, también es quien comunica y plantea un protocolo básico de atención bucodental, salvo en $34 \%$ de las pacientes que no recibieron dicha información. Asimismo, en $10 \%$ de los casos los médicos generales se encargaron de notificar y en $8 \%$ fueron las enfermeras. Al respecto, el trabajo de Taichman et al. (2018) reportó que el oncólogo y las enfermeras son quienes, principalmente, cuestionan a los pacientes sobre su salud bucal; en ese sentido, en dicha investigación se señaló que $46 \%$ de los encuestados 


\section{Revista Iberoamericana de las Ciencias Sociales y Humanísticas}

ISSN: $2395-7972$

no tuvo una visita con el odontólogo desde el diagnóstico de cáncer debido a que no forma parte del protocolo. Por tanto, no hay seguimiento buco-dental y, en consecuencia, no existe un diálogo sobre su condición.

Asimismo, en los hallazgos de esta investigación se encontró que, si bien las pacientes saben que reciben quimioterapia, $82 \%$ desconoce el nombre de los fármacos. Es posible que esto se relacione con el intervalo de tiempo en que se ha recibido la quimioterapia debido a que $54 \%$ presenta un periodo menor a un año con la terapia farmacológica. Aunque las pacientes no reportaron mucositis oral al momento de responder las cédulas, es necesario tomar en consideración esta situación, ya que cuanto más se prolongue la quimioterapia existen mayores posibilidades para que se presenten lesiones bucales. En este sentido, el estudio realizado por Sonis (2012) reporta sobre ciertos fármacos que provocan mucositis en $20 \%$ de los pacientes en el primer ciclo de quimioterapia y para el segundo ciclo con los mismos fármacos la mucositis se incrementa en $70 \%$ de los casos. Por lo tanto, se debe hacer énfasis en el acompañamiento durante el proceso de la enfermedad, así como en la creación de un plan de acción multidisciplinario ante la creciente demanda de atención.

En la mucositis oral el tipo de cepillo, enjuague y pasta dental no son los convencionales, ya que se requiere de una implementación particular de acuerdo con la condición del paciente. Además, la técnica de cepillado debe adaptarse según el estado bucal y dental particular; por ejemplo, el cepillo deberá ser ultrasuave (semejante al de uso posterior a cirugías), así como los enjuagues libres de alcohol o con bencidamina, y pasta dental de preferencia antiplaca.

Los resultados presentados sugieren la necesidad de acompañamiento en las pacientes y la apertura al diálogo acerca del conocimiento de las prioridades según el diagnóstico bucal. En este sentido, es imperativo el trabajo en equipo multidisciplinario bajo un marco de diálogo que favorezca la relación médico-paciente, pues solo de esta manera será auténtico el acompañamiento del paciente en su condición médica (Joshi, 2010).

Aunque $54 \%$ de las pacientes reportaron que no presentaban úlceras bucales, $46 \%$ sufría de úlceras leves o muy severas, las cuales si bien no provocaron la interrupción de la quimioterapia, sí impactaron en la calidad de vida porque mermaron la posibilidad de comer, beber y comunicarse. Es esencial, por ende, el diálogo con dichas pacientes para 


\section{Revista Iberoamericana \\ de las Ciencias Sociales y Humanísticas}

ISSN: 2395 - 7972

aminorar los síntomas de la mucositis oral, evitar los agravantes y mejorar su calidad de vida bucodental.

De acuerdo con Gadamer (2001), el diálogo es parte del tratamiento, el cual es inseparable de la comprensión del paciente para desembocar en la recuperación. Por ello, es vital comprender el sentir y pensar de las pacientes.

En concordancia con esta idea, Taichman et al. (2018) señalan el especial interés en la comunicación entre el paciente y los proveedores de salud (oncólogo, enfermera, dentista, higienista dental y médicos de atención primaria), ya que las pacientes con cáncer de mama deben recibir atención profesional preventiva y de cuidado bucal.

En este contexto, existen medidas de apoyo médico para mejorar los síntomas de las úlceras, con las cuales es posible lograr que las necesidades básicas de todo ser humano se lleven a cabo en las mejores condiciones, y no repercutan en otras dimensiones. Téngase en cuenta que la interacción de las dimensiones es estrecha, y cuando una de ellas se ve afectada el resto también se daña.

Por otra parte, vale indicar que la mitad de los pacientes estima la confianza y el servicio que ofrece una institución dedicada y especializada en el manejo del cáncer de mama, de ahí que la consideren como la primera instancia médica de asistencia en caso de presentar úlceras bucales por efecto secundario de la quimioterapia. Esto significa que son pocas las personas $(26 \%$ ) que acuden al dentista para tratar dicho problema, aun cuando es el especialista en la cavidad bucal y en sus estructuras.

Por otra parte, al considerarse una muestra aleatoria, se puede indicar que $54 \%$ de las pacientes reportaron que tenían menos de un año con la terapia farmacológica, de ahí que no estén familiarizadas con el nombre de los fármacos. Asimismo, no indicaron la presencia de mucositis oral en el momento de responder las cédulas, aunque cabe recordar que dicha enfermedad es más latente cuando el tratamiento con quimioterapia se prolonga. 


\section{Revista Iberoamericana}

de las Ciencias Sociales y Humanísticas

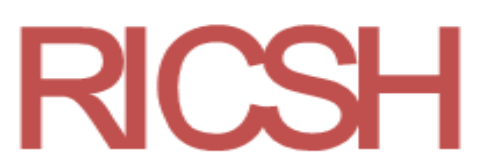

ISSN: 2395 - 7972

\section{Conclusiones}

Los resultados recabados en la presente investigación permiten extraer las siguientes conclusiones: es casi nulo el diálogo entre el odontólogo y las pacientes que reciben quimioterapia por cáncer de mama, lo cual se debe a la ausencia de dicho profesional en el equipo multidisciplinario que trata esa enfermedad. De hecho, se puede asegurar que en la mayoría de los casos las manifestaciones bucales secundarias de la quimioterapia son atendidas por el oncólogo y las enfermeras, de ahí que la atención se limite a medidas de higiene bucal general.

Por otra parte, cabe resaltar que la importancia de incorporar en la praxis médica a la hermenéutica filosófica, desde una perspectiva gadameriana, radica en el planteamiento de centrar el diálogo durante el tratamiento para escuchar al paciente, con lo cual se da apertura a la comprensión del otro y, por tanto, al conocimiento de la enfermedad y al logro de un mínimo equilibrio en la salud.

Asimismo, los reportes permiten valorar la participación del oncólogo, odontólogo y personal de la salud desde la mirada del paciente. Así, la cédula se presenta como un instrumento indispensable para reflexionar sobre la praxis médica y aproximarse al sentir y pensar del paciente sobre su enfermedad. La comunicación entre los equipos oncológico y odontológico es esencial para la seguridad del paciente, así como el diálogo entre el paciente y el odontólogo, ya que la hiposalivación generada por la quimioterapia puede incrementar la gingivitis y la caries dental, así como las alteraciones en el sentido del gusto. La evaluación bucodental, entonces, es necesaria antes de iniciar la quimioterapia para identificar las necesidades que puedan incrementar el riesgo o gravedad de las enfermedades orales durante el tratamiento contra el cáncer de mama.

Aunado a esto, se debe prever que las pacientes con terapia antiestrogénica pueden presentar elevados niveles de depresión, síntomas músculo-esqueléticos y fatiga, de tal forma que impactan su habilidad para mantener una óptima salud bucal. Por ello, en la medida que exista un diálogo entre el personal de la salud y los pacientes, estos últimos podrán tener conocimiento sobre los efectos secundarios de los medicamentos para crear un plan de acción que permita aminorar los síntomas de la mucositis oral y otros padecimientos.

El odontólogo, en síntesis, al ser un profesional de la salud bucal debe atender diversos aspectos en el paciente, como el conocimiento del cuadro clínico. Por eso, las autoras de este trabajo proponen establecer redes de trabajo disciplinario y 
Revista Iberoamericana

de las Ciencias Sociales y

Humanísticas

ISSN: $2395-7972$

transdisciplinario con odontólogos activos y en formación para establecer indicadores que permitan detectar aspectos relevantes en el paciente durante su seguimiento y acompañamiento con el fin de promover su calidad de vida bucal.

\section{Futuras líneas de investigación}

En este trabajo se visibilizó la dinámica en la relación médica odontólogo-paciente respecto al desconocimiento de los últimos sobre la mucositis oral generada por quimioterapia. En tal sentido, vale acotar que si bien cada día surgen grupos de fármacos más eficientes para el manejo del cáncer de mama, también algunos tienen más toxicidad que provoca mayor agresión a la mucosa oral. Por eso, resultan esenciales estudios que se enfoquen en escuchar las voces de las pacientes sobre la comprensión de su condición de salud (resultado reportado por el paciente, PROM), las cuales pueden ser distintas a la del médico e incluso más distantes si no existió el diálogo, sino solo el intercambio unilateral de información.

Otro aspecto relevante de carácter metodológico que se puede considerar en futuras investigaciones es la selección de una muestra por conveniencia, ya que más de la mitad de las pacientes entrevistadas tenían menos de un año con la terapia farmacológica, motivo por el cual no reportaron la mucositis oral al momento de responder las cédulas. Al respecto, téngase en cuenta que dicha enfermedad es más usual cuando el tratamiento es más prolongado. 
Revista Iberoamericana

de las Ciencias Sociales y

Humanísticas

ISSN: $2395-7972$

\section{Referencias}

Austgard, K. (2012). Doing it the Gadamerian way-using philosophical hermeneutics as a methodological approach in nursing science. Scandinavian Journal of Caring Sciences, 26(4), 829-834. Doi: https://doi/pdf/10.1111/j.1471-6712.2012.00993.x

Ávila, M. J. (2017). La deshumanización en medicina. Desde la formación al ejercicio profesional. $\quad$ Iatreia, $216-229$. https://dx.doi.org/10.17533/udea.iatreia.v30n2a11

Barroso-Sousa, R., Santana, I. A., Testa, L., De Melo Gagliato, D. and Mano, M. S. (2013). Biological therapies in breast cancer: common toxicities and management strategies. The Breast, 22(6), 1009-1018. Doi: https://doi.org/10.1016/j.breast.2013.09.009

Basch, E., Reeve, B. B., Mitchell, S. A., Clauser, S. B., Minasian, L. M., Dueck, A. C. and Bruner, D. W. (2014). Development of the National Cancer Institute's patientreported outcomes version of the common terminology criteria for adverse events (PRO-CTCAE). Journal of the National Cancer Institute, 106(9). Doi: https://doi.org/10.1093/jnci/dju244.

Beuchot, M. (2016). Hechos e interpretaciones. México: Fondo de Cultura Económica.

Ceriani, J. (2017). La práctica médica en la era tecnológica. Arch Argent Pediatr, 115(2), 106-107.

Fleury, S. y Lana da Costa, Z. (2004). Qualidade de vida e saúde: aspectos conceituais e metodolügicos. Cad Saúde Pública, 20(2), 580-588.

Gadamer, H. G. (1993). Verdad y método (tomo II). Salamanca: Sígueme.

Gadamer, H. G. (2001). El estado oculto de la salud. Barcelona: Gedisa.

Gadamer, H. G. (2005). Verdad y método (tomo I). España: Sígueme.

Jensen, S. B., Mouridsen, H. T., Bergmann, O. J., Reibel, J., Brünner, N. and Nauntofte, B. (2008). Oral mucosal lesions, microbial changes, and taste disturbances induced by adjuvant chemotherapy in breast cancer patients. Oral Surgery, Oral Medicine, Oral Pathology and Oral Radiology, 106(2), 217-226. Doi: https://doi.org/10.1016/j.tripleo.2008.04.003

Joshi, V. K. (2010). Dental treatment planning and management for the mouth cancer patient. Oral $\quad$ Oncology, 46(6), 475-479. Doi: https://doi.org/10.1016/j.oraloncology.2010.03.010 


\section{Revista Iberoamericana}

de las Ciencias Sociales y Humanísticas

ISSN: $2395-7972$

NCI- PRO-CTCAETM ITEMS-SPANISH Item Library (version 1). Retrieved from https://healthcaredelivery.cancer.gov/pro-ctcae/pro-ctcae_spanish.pdf

Posada, S. R. (2009). Hermenéutica y medicina. CES Medicina, 23(1), 103-113. Doi: https://doi.org/10.1002/1097-0142(20010215)

Seiler, S., Kosse, J., Loibl, S. and Jackisch, C. (2014). Adverse event management of oral mucositis in patients with breast cancer. Breast Care, 9(4), 232-237.

Senkus, E., Kyriakides, S., Penault-Llorca, F., Poortmans, P., Thompson, A., Zackrisson, S. and ESMO Guidelines Working Group. (2013). Primary breast cancer: ESMO Clinical Practice Guidelines for diagnosis, treatment and follow-up. Annals of oncology, 24(suppl_6), vi7-vi23.

Sonis, S. T. (2012). Oral Mucositis. Pocket books for cancer supportive care. Boston: Springer Healthcare.

Taichman, L. S., Gomez, G. and Inglehart, M. R. (2015). Oral health-related complications of breast cancer treatment: assessing dental hygienists knowledge and professional practice. American Dental HygienistsAssociation, 89(2), 22-37.

Taichman, L. S., Poznack, C. H. and Inglehart, M. R. (2018). Oral health-related concerns, behavior, and communication with health care providers of patients with breast cancer: impact of different treatments. Special Care in Dentistry, 38(1), 36-45. Doi: https://doi.org/10.1111/scd.12266

Viveros, E. F. (2019). El diálogo como fusión de horizontes en la comprensión hermenéutica de Gadamer. Perseitas, 7(2), 341-354. Doi: https://doi.org/10.21501/23461780.3293

Walsh, L. J. (2010). Clinical assessment and management of the oral environment in the oncology patient. Australian Dental Journal, 55(1), 66-77. 


\section{Revista Iberoamericana de las Ciencias Sociales y Humanísticas}

ISSN: 2395 - 7972

\begin{tabular}{|c|c|}
\hline Rol de Contribución & Autor (es) \\
\hline Conceptualización & Alma Alicia Benítez Pérez y Alejandra Sosa Gómez. (Iguales) \\
\hline Metodología & Alejandra Sosa Gómez y Alma Alicia Benítez Pérez (Iguales) \\
\hline Software & Alejandra Sosa Gómez y Alma Alicia Benítez Pérez (Iguales) \\
\hline Validación & Alma Alicia Benítez Pérez y Alejandra Sosa Gómez. (Iguales) \\
\hline Análisis Formal & $\begin{array}{l}\text { Análisis cualitativo. Alejandra Sosa Gómez y Alma Alicia } \\
\text { Benítez Pérez. (Iguales) }\end{array}$ \\
\hline Investigación & Alma Alicia Benítez Pérez y Alejandra Sosa Gómez. (Iguales) \\
\hline Recursos & Alejandra Sosa Gómez y Alma Alicia Benítez Pérez (Iguales) \\
\hline Curación de datos & Alejandra Sosa Gómez y Alma Alicia Benítez Pérez (Iguales) \\
\hline $\begin{array}{l}\text { Escritura - Preparación del } \\
\text { borrador original }\end{array}$ & $\begin{array}{l}\text { Alejandra Sosa Gómez y Alma Alicia Benítez Pérez. (Iguales), } \\
\text { María Elena Michelle Pérez Medina (que apoya) }\end{array}$ \\
\hline $\begin{array}{l}\text { Escritura - Revisión y } \\
\text { edición }\end{array}$ & Alma Alicia Benítez Pérez y Alejandra Sosa Gómez (Iguales) \\
\hline Visualización & Alejandra Sosa Gómez y Alma Alicia Benítez Pérez (Iguales) \\
\hline Supervisión & Alma Alicia Benítez Pérez y Alejandra Sosa Gómez (Iguales) \\
\hline Administración de Proyectos & Alma Alicia Benítez Pérez y Alejandra Sosa Gómez. (Iguales) \\
\hline Adquisición de fondos & Alejandra Sosa Gómez y Alma Alicia Benítez Pérez (Iguales) \\
\hline
\end{tabular}

